\title{
La lecture numérique entre l'interactivité et la gestualité
}

\author{
Dalia Metawe* \\ dalia.metawe@yahoo.com
}

\section{Résumé}

Le concept traditionnel de lecture incite les lecteurs à lire systématiquement des textes continus du début à la fin. Cette image peut être modifiée lors de la lecture sur un écran d'ordinateur, une tablette, une liseuse ou un téléphone portable. Avec l'avènement des ordinateurs multimédias, en particulier l'apparition de l'Internet, les caractéristiques et le statut du texte numérique ont subi des changements fondamentaux, qui ont complètement changé notre rapport à la lecture. Le numérique devient un outil créatif actif et établit une relation mutuelle entre le système informatique et ses utilisateurs. Par conséquent, au cours des deux dernières décennies, la pratique de la lecture numérique est devenue un courant dominant, en particulier chez les jeunes. Cette étude traitera de la particularité de la pratique de lecture et le statut des lecteurs sur support numérique. La différence entre la lecture numérique et la lecture traditionnelle sous forme imprimée sera abordée. Nous examinerons si cette lecture numérique a un style spécifique qui permet aux lecteurs d'intervenir dans l'œuvre, et si cette nouvelle méthode de lecture en ligne affecte la construction du sens de l'œuvre.

Mots-clés : lecture numérique, style, interactivité, manipulation, gestualité.

\footnotetext{
* Pr. adjoint en linguistique - Faculté des lettres - Département de français- Université de Menoufia.
}

(La lecture numérique entre l'interactivité ...)Dr. Dalia Metawe 


\section{$\underline{\text { La lecture numérique entre l'interactivité et la gestualité }}$}

Le concept traditionnel de lecture incite les lecteurs à lire systématiquement des textes continus du début à la fin. Cette image peut être modifiée lors de la lecture sur un écran d'ordinateur, une tablette, une liseuse ou un téléphone portable. Au cours des deux dernières décennies, la pratique de la lecture numérique est devenue un courant dominant, en particulier chez les jeunes. À cet égard, se posent certaines questions : quelles sont les spécificités qui caractérisent la lecture numérique? L'existence d'appareils électroniques pour la lecture (comme les PC, les liseuses ou les tablettes) changera-t-elle les circonstances, l'environnement et les besoins qui provoquent la lecture? La lecture numérique nécessite-t-elle de nouvelles connaissances et/ou compétences des lecteurs? Le style du texte numérique est-il différent du texte imprimé? Et enfin, la nature du texte lu modifiera-t-elle la pratique de la lecture?

Pour tenter de répondre à ces questions, cette étude traitera de la particularité de la pratique de lecture et le statut des lecteurs sur support numérique. La différence entre la lecture numérique et la lecture traditionnelle sous forme imprimée sera abordée. Nous examinerons si cette lecture numérique a un style spécifique qui permet aux lecteurs d'intervenir dans l'œuvre, et si cette nouvelle méthode de lecture en ligne affecte la construction du sens de l'œuvre.

(La lecture numérique entre l'interactivité ...)Dr. Dalia Metawe 
Avant d'aborder notre sujet, qu'entend-on par «lecture numérique »? Ce terme fait référence à l'activité de lecture de textes écrits (qui peuvent être accompagnés d'images fixes ou d'illustrations animées) au moyen d'appareils numériques (ordinateurs, tablettes, smartphones, bornes d'information ou autres) (J. F. Rouet, 2016 ; E. Chandelier, 2019). Notons que la lecture numérique, appelée parfois «lecture sur écran», comprend plusieurs types de lecture : la lecture hypertextuelle, la lecture de pages web et la lecture d'œuvres numériques. Dans ce travail, nous nous concentrerons sur les activités de lecture d'œuvres littéraires numériques. Qu'il s'agisse d'un message texte (SMS), d'une page Web ou d'un roman en ligne, les lecteurs peuvent accéder au texte numérique grâce à une combinaison d'exploration visuelle (comme des listes ou des menus) et des actions explicites (comme la formulation de requêtes ou la sélectionne des liens).

\section{Texte et lecture sur support numérique}

Avec l'avènement des ordinateurs multimédias, en particulier l'apparition de l'Internet, le statut et les caractéristiques du texte numérique ont subi des changements fondamentaux, qui ont complètement changé notre rapport à la lecture. Le numérique, domaine en constante évolution, est devenu un outil de création marqué et a établi une relation mutuelle entre le système informatique et ses utilisateurs. Par

(La lecture numérique entre l'interactivité ...)Dr. Dalia Metawe 
conséquent, la plupart de la création littéraire, artistique et publicitaire numérique repose sur les gestes de manipulation des lecteurs/internautes du contenu de l'écran et/ou des textes euxmêmes.

Pour comprendre la particularité de la lecture numérique, nous devons d'abord vérifier la particularité du texte numérique, la situation qui stimule les activités de lecture, les connaissances et les compétences des lecteurs. Force est de constater que l'émergence du Web 2.0 a provoqué de réels changements dans le concept fondateur d'écriture littéraire et de texte. Grâce à cette technologie, les auteurs connectés à Internet peuvent créer des textes numériques qui ne sont pas seulement des textes lisibles, mais aussi des textes mouvants et manipulables. Ils utilisent les potentiels du monde numérique comme apports stylistiques pour élaborer une production littéraire écrite et parue exclusivement sur le web ou les applications informatiques.

De nouvelles formes littéraires hypermédiatiques, dynamiques et multi-codes sont donc apparues en ligne. Malgré les différences d'objectifs, de formats et de méthodes de production, ces œuvres numériques partagent généralement une structure composite entrelaçant avec la forme textuelle de l'œuvre, d'illustrations, d'animations et du contenu audio, pour former ensemble la structure globale de l'œuvre. L'affichage hypermédiatique de cette création numérique conduira à une

(La lecture numérique entre l'interactivité ...)Dr. Dalia Metawe 
lecture dynamique, dans laquelle les lecteurs jouent un rôle majeur. Ainsi, la nature de la production numérique et les supports informatiques déterminent l'acte spécifique de lecture en ligne en fonction des activités du lecteur à lesquelles nous reviendrons. Abordons à présent la technologie hypertexte et explorons son rôle dans le changement de l'acte de lecture sur support numérique.

\section{La technologie hypertexte}

La lecture sur Internet est basée sur l'hypertexte, un terme conçue en 1965 par le philosophe et pionnier de l'informatique Ted Nelson. L'hypertexte est l'organisation du texte, qui peut être parcouru de plusieurs façons en cliquant sur des liens. Étudions à présent comment cette technologie peut modifier l'acte de la lecture numérique. Compte tenu du fait que l'hypertexte utilise des liens hypertextes pour relier plusieurs fragments de différentes longueurs (Gaëlle Debeaux, 2019), A. Saemmer signale que l'hypertexte n'associe pas seulement le texte de départ qu'elle propose d'appeler «texte géniteur» à un ou plusieurs autres textes appelés «textes reliés». Il est caractérisé également par une manipulabilité qui implique un enchaînement spécifique de gestes effectué par le lecteur (2011 : 48).

D'un point de vue théorique, A. Saemmer a expliqué que l'hypertexte constitue un "couplage pluricode » entre un signe

(La lecture numérique entre l'interactivité ...)Dr. Dalia Metawe 
linguistique et un signe «iconique » (un symbole tel que les traits de soulignement) et que la relation entre le «signifiant graphique » et le signifié «manipulabilité » est conforme aux conventions établies: en fonctionnement, le lecteur/utilisateur manipule l'hypertexte «cliquable » à travers une série de gestes exécutés dans un but précis. Après avoir effectué les gestes de manipulation, les attentes du lecteur vis-à-vis de la cible sont plus ou moins satisfaites par le résultat effectif de la manipulation (2011 : 48). Saemmer adopte à cet égard une approche de la lecture considérée comme « une rencontre entre l'anticipation des pratiques par le texte numérique, et les imaginaires individuels et socialement partagés du lecteur qui préfigure et actualise ces anticipations » (2015: 63). En d'autres termes, sa méthode consiste à comparer les effets attendus de la lecture de texte numérique (Saemmer les appelle «figures de la lecture») avec ce que le lecteur espère réaliser en lecture numérique. Selon Saemmer et d'autres chercheurs, dont Bouchardon, ils sont appelés «horizons d'attente » ou «figurations » (Ibid.: 15).

La plupart des créations littéraires numériques interactives sont basées sur la technique de l'hypertexte. Par conséquent, cette production littéraire en ligne a modifié l'acte de lecture linéaire traditionnel en fournissant une méthode de lecture non-linéaire qui permet à chaque lecteur de suivre un parcours singulier selon les hyperliens qu'il active. Notons que «Afternoon, a story»,

(La lecture numérique entre l'interactivité ...)Dr. Dalia Metawe 
une œuvre numérique de l'auteur américain Michael Joyce écrite en 1987 et publiée sur disquette par la compagnie Eastgate Systems en 1990, est considérée comme l'une des premières créations interactives hypertextuelles. Eastgate a continué de publier l'œuvre dans les années 2010, et l'a distribué sur des clés USB.

Le texte hypertextuel dépasse donc la contrainte et la limite de la linéarité du texte imprimé et le sens imposé par la matérialité du livre papier. Dans ce contexte, les écrivains de l'hypertexte fictionnel créent des histoires en ligne selon double contrainte : non-linéarité et fragmentation de l'histoire. Bien que le vocable «hypertexte » soit apparu récemment, l'idée d'écrire des récits non-linéaires est en effet ancienne. Mentionnons par exemple Tristram Shandy de Laurence Sterne (1760), Cent mille milliards de poèmes et Un conte à votre façon de R. Queneau (1961, 1967) et Le grand incendie de Londres de J. Roubaud (1989). Pour désigner la littérature dans laquelle le lecteur fait un effort pour parcourir le texte, Espen J. Aarseth a inventé en 1997 le terme «littérature ergodique » (Cf., E. J. Aarseth, 1997). Cependant, les ordinateurs utilisent des liens hypertextes ou des hyperliens pour automatiser et programmer ce travail de lecture non linéaire. Cette technologie complexe de génération de textes et de documents audiovisuels ne peut pas être produite sous forme papier. Par ailleurs, la fragmentation des fictions hypertextuelles

(La lecture numérique entre l'interactivité ...)Dr. Dalia Metawe 
a suscité des recherches intéressantes, car elle semble constituer l'une des principales caractéristiques de la poétique narrative dans l'environnement numérique ( $C f$., entre autres, R. Audet \& S. Brousseau, $2011: 10)$.

Dans un récit hypertextuel, le lecteur/internaute navigue d'un lien à l'autre selon son choix et son acte individuel. C'est lui qui décide sa modalité de lecture. La lecture d'une œuvre hypertextuelle fait donc toujours appel à la subjectivité du lecteur/internaute. Serge Bouchardon a expliqué le dynamisme de ce parcours: «les liens peuvent en effet être statiques mais ils peuvent également être dynamiques : si le lecteur clique à plusieurs reprises sur un même lien hypertexte, soit au cours d'un même parcours de lecture, soit lors d'une lecture ultérieure, il ne verra pas forcément le même fragment textuel s'afficher » (2007). Ainsi l'auteur de l'hypertexte prédéfinit un «lecteur modèle » qui doit relier les fragments textuels des œuvres. Quant au lecteur, il utilise ses compétences pour redonner du sens au texte en construisant son propre hypertexte. La particularité de ce type de lecture est que devant l'écran de l'ordinateur, le lecteur/internaute équipé d'une souris ou d'un autre périphérique d'entrée est en état d'attente pour voir comment le programme réagit à ses demandes qu'il effectue à son tour comme réaction aux suggestions venues du programme. Ainsi, selon J. Clément, en plus d'activer de simples liens hypertextes, il existe tout un

(La lecture numérique entre l'interactivité ...)Dr. Dalia Metawe 
ensemble d'opérations possibles qui ont autant d'effets que de surprises et de découvertes. La réponse de la machine peut être générée aléatoirement, renforçant ainsi les effets inattendus, mais ce caractère aléatoire n'est pas nécessaire $(C f$., J. Clément, $2011: 73)$.

J. Clément a mis en évidence la dimension aléatoire de presque toutes les œuvres numériques programmées. Selon lui, cet aspect contrôle la structure de l'œuvre, et la structure de l'œuvre peut être limitée à une génération automatique. Il peut également fixer la réponse aux demandes volontaires ou involontaires des lecteurs/internautes ou de leur environnement. Dans le monde numérique, l'aléatoire devient donc un procédé de création qui constitue l'une des poéticités de la littérature numérique. Cependant, Clément a souligné que l'auteur ne peut pas contrôler entièrement la création : l'auteur ne peut contrôler que le programme, mais pas l'œuvre qu'il produit, car dans les œuvres aléatoires, l'auteur remet la machine en place après avoir démarré le programme (Ibid. : 75).

C'est dans cette perspective que l'hypertexte est interprété comme une «interactivité ». Le lecteur peut interagir avec des hyperliens et modifier le sens du texte, et dans ce cas la modification doit être comprise comme équivalent à l'écriture. Notons que dans le discours numérique, du fait de la démocratisation des outils d'écriture et de l'utilisation des

(La lecture numérique entre l'interactivité ...)Dr. Dalia Metawe 
publications en ligne, les auteurs et les lecteurs ont de moins en moins de fonctions sociales séparées. Les lecteurs/ internautes peuvent désormais dialoguer avec les auteurs via leurs sites Web d'auteurs ou des ateliers de rédaction en ligne et proposer des idées de travaux en cours. Cette interaction offre aux lecteurs plus d'opportunités de pratiquer l'écriture créative en ligne $(C f$., Saemmer, 2011: 67). Lorsque la lecture devient une véritable performance sur laquelle nous reviendrons, elle offre également à chaque lecteur une expérience personnelle unique, en particulier lorsqu'il y a interactivité. Chacun maitrisera les fragments de sens qu'il a assemblés pour construire son propre sens ou se développer dans l'histoire selon ses propres préférences. Le texte est donc un sujet expérimental sur sa position dans l'espace et son «traitement graphique » $(C f$., Clara Henry, $2018: 23$ ).

L'hypertexte appartenant à l'informatique est ainsi ouvert, fragmenté, et propice à de multiples actes de lecture, et offre au lecteur/usager une grande subjectivité dans son parcours de lecture et d'interprétation. Saemmer va en effet au delà de cette réflexion en affirmant, d'un point de vue stylistique, que l'hypertexte n'est pas un simple lien entre des nœuds d'informations, mais dans certains cas, il peut aussi agir comme une «figure » et générer une nouvelle expérience de lecture. Les lecteurs de ce type pourront développer une relation réflexive au

(La lecture numérique entre l'interactivité ...)Dr. Dalia Metawe 
potentiel sémantique du texte numérique, et commencer à éprouver un nouveau type de plaisir textuel, qui va au-delà d'un clic frénétique bref et du zap démesuré ( $C f$., Saemmer, 2011: 66). De même, Emily Golson a indiqué que les associations «imprévisibles» entre «texte géniteur» et «textes reliés», peuvent être considérées comme des «figures de style»: contrairement aux mises en relation «littérales », elles impliquent des actes de «médiation » par le lecteur (1999:66).

\section{La dimension multimédia et l'interactivité avec le $\underline{\text { lecteur/internaute }}$}

Nous avons étudié la technique des hyperliens du web en mettant en relief les modifications qu'elle a apportées à l'acte de lecture en ligne. Néanmoins, les récits hypertextes purs sont actuellement de plus en plus rares sur Internet. L'hypertextualité n'est pas le seul potentiel de l'informatique qui a modifié la lecture numérique dans le domaine de la littérature électronique : la dimension multimédia et d'autres formes d'interactivité ont apporté de l'innovation à la lecture sur support numérique : « $L a$ tendance actuelle est aux hypermédias animés en ligne qui exploitent conjointement l'affichage dynamique du texte et la dimension multimédia et mettent l'accent sur l'interactivité avec un lecteur »(S. Bouchardon, 2012 a : 13-14 ; 2014 : 90). Pour sa part, Philippe Bootz a affirmé que toutes les œuvres littéraires numériques ont tendance à être hypertextuelles, génératives et

(La lecture numérique entre l'interactivité ...)Dr. Dalia Metawe 
animées (2011 : 206). En conséquence, l'accent est désormais mis sur l'animation plutôt que sur l'hypertexte ou la dimension générative.

L'animation multimédia et l'interactivité, sont les principales tendances de la littérature numérique en France, aux Etats-Unis et au Canada. Ces tendances concernent les œuvres destinées à être lues sur Internet surtout avec l'émergence des réseaux sociaux (notamment Facebook, Twitter et Snapchat) qui ont offert aux auteurs de nouvelles fenêtres et de nouveaux terrains de leurs créations numériques. L'hypertexte ne suscite plus l'intérêt des auteurs connectés au réseau, mais l'hypermédia en ligne animé. En fait, avec l'évolution de plus en plus puissante d'ordinateurs, ils tentent désormais de transférer et de mettre en question la vidéo, l'interaction et toutes les nouvelles fonctionnalités offertes par Internet ( $C f$., C. Henry, $2018: 13$ ).

Sur support numérique, le texte linguistique est caractérisé par sa forme et sa couleur, en particulier par son animation et sa manipulabilité. Ces textes animés et manipulables se composent de «couplage pluricode», pour reprendre l'expression d'A. Saemmer (2011: 48), constituant des combinaisons spécifiques de différents systèmes sémiotiques : textes, images, animations, vidéos, sons. En d'autres termes, sur le même support actif se trouvent réunis un signe linguistique, par exemple un mot, et un signe «iconique » qui exprime des référents d'expérience à

(La lecture numérique entre l'interactivité ...)Dr. Dalia Metawe 
travers sa structure perceptible, par exemple un mouvement, et/ou un enchaînement de gestes de manipulation effectué dans une attente (Ibid.: 50). Ainsi l'iconicité (entendue comme la représentation visuelle d'un signe) caractérise le texte numérique qui n'est plus un texte lisible, mais un texte à visualiser et à manipuler. Ce concept théorise la partie sensible du sens dans le texte numérique, qui s'exprime par les manipulations, les mouvements, les gestes du lecteur.

Par conséquent, l'animation textuelle devient un lieu d'expérimentation poétique, car l'accent est mis sur les signes pour altérer ce à quoi ils font référence en donnant le lecteur/internaute l'occasion de construire lui-même sa propre interprétation de l'œuvre. Citons par exemple le poème animé de Jean-Pierre Balpe, Plus tard, paru en ligne (http://www.balpe.name/Plus-tard), en Juillet 2020, où les signes employés proviennent de différents systèmes sémiotiques (linguistique, graphique, animation, sons). Les lettres et les mots subissent une conversion temporelle à l'écran : modification, déplacement, morphing. Ils se transforment sur l'écran de l'ordinateur en images animées, comme le montrent successivement les figures (1), (2) et (3). Le lecteur entend des voix chaotiques d'hommes et de femmes récitant le poème, et à la fin de cette création numérique, le poème apparaît progressivement à l'écran, comme le désigne la figure (4). La

(La lecture numérique entre l'interactivité ...)Dr. Dalia Metawe 


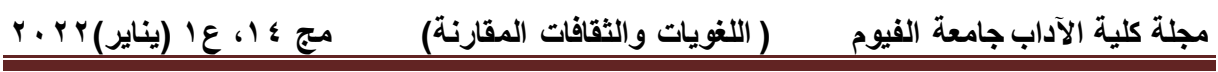

figure (5) indique l'image finale du poème affichée à l'écran avec un fond noir :

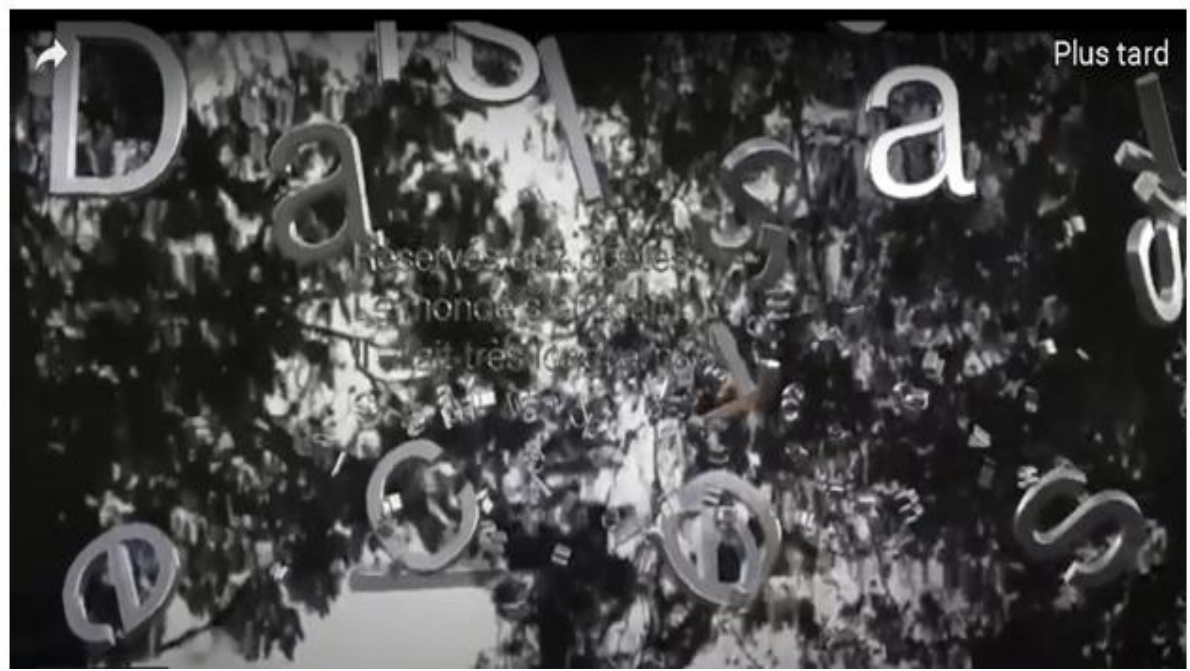

Figure (1) : capture-écran, Plus Tard de J.-P. Balpe (2020).

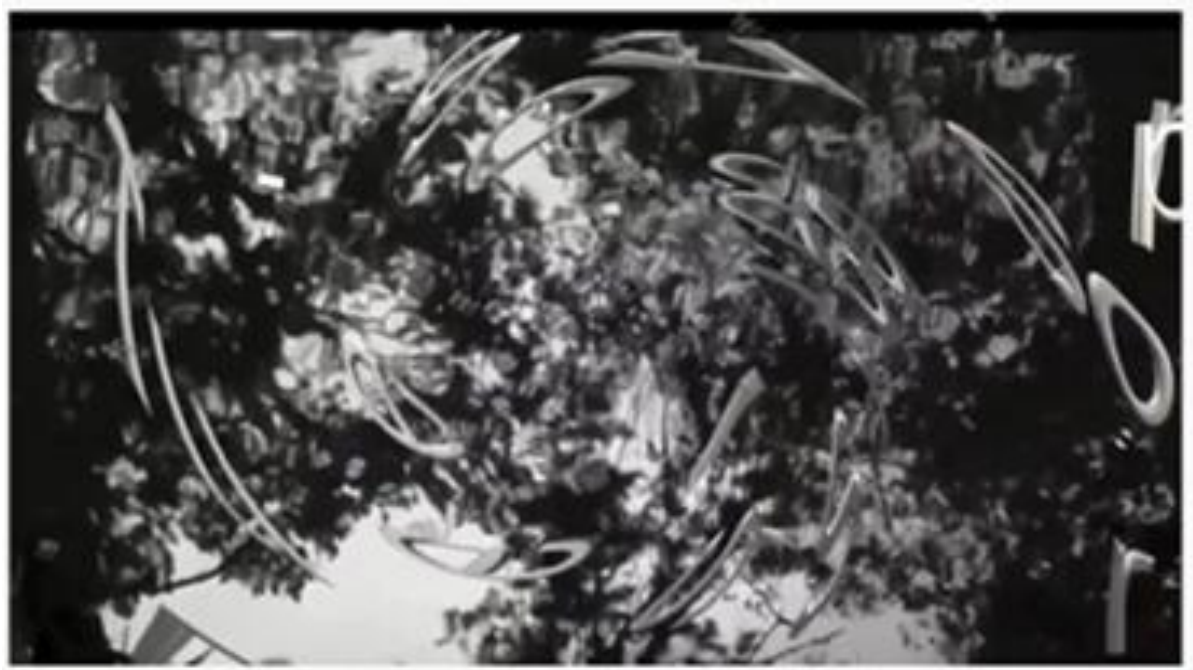

Figure (2) : capture-écran, Plus Tard de J.-P. Balpe (2020).

(La lecture numérique entre l'interactivité ...)Dr. Dalia Metawe 


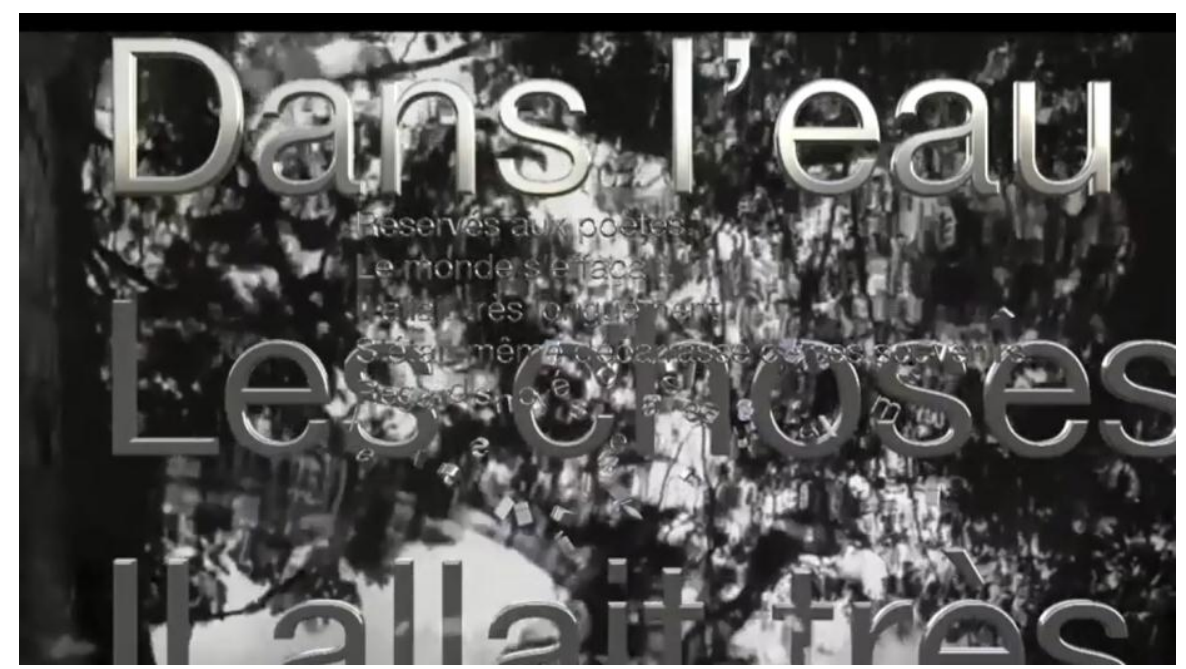

Figure (3) : capture-écran, Plus Tard de J.-P. Balpe (2020).

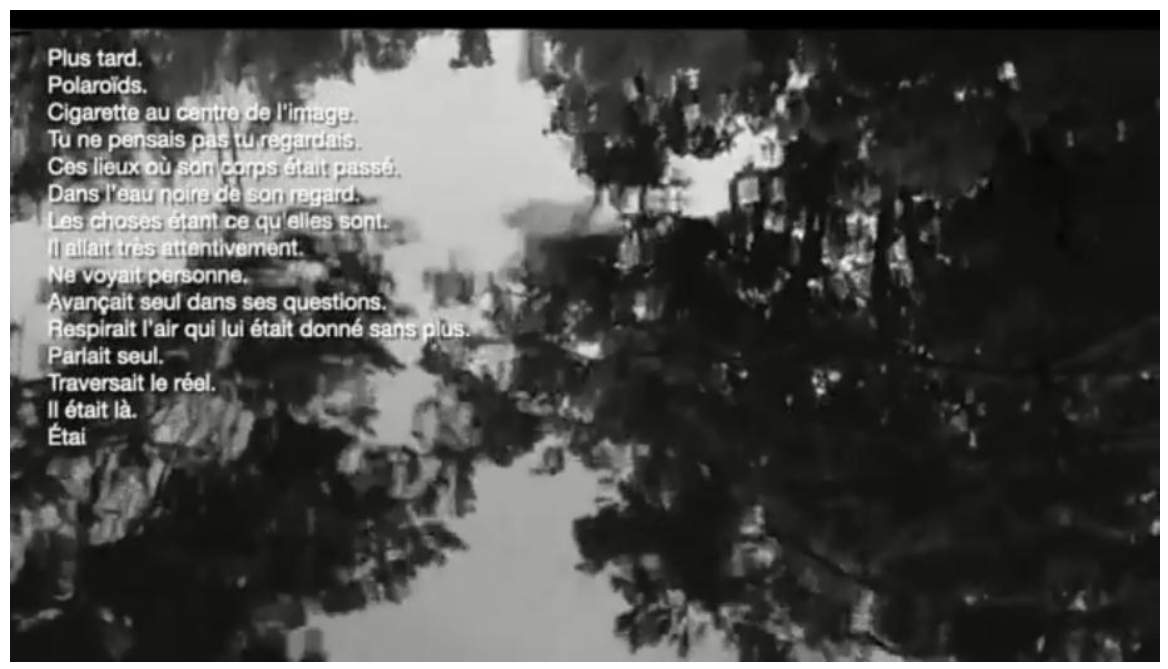

Figure (4) : capture-écran, Plus Tard de J.-P. Balpe (2020).

(La lecture numérique entre l'interactivité ...)Dr. Dalia Metawe 
Figure (5) : l'image finale du poème

Notons que les hyperliens et les animations mettent en évidence la participation physique du lecteur à l'œuvre numérique : lorsqu'un lecteur/usager active un lien hypertexte, il clique physiquement sur ce lien avec la souris. L'animation de la création numérique est, pour sa part, réalisée de deux manières proposées par l'auteur en utilisant les deux concepts «d'unité sémiotique de la manipulation» et « d'unité sémiotique de mouvement ». Selon le point de vue d'A. Saemmer, ces concepts nécessitent des «référents d'expériences », c'est-à-dire les pratiques et réactions des interacteurs, et produisent ce que Saemmer appelle des «simulacres de référents » en assemblant des gestes physiques, du texte et des icônes (Saemmer, 2011: 28).

Ces dimensions dynamiques contribuent à donner à la lecture numérique un style dynamique particulier et un caractère

(La lecture numérique entre l'interactivité ...)Dr. Dalia Metawe 
plurisémiotique et synesthésique qui active des modalités de perceptions différentes venues de domaines sensoriels différents. Les lecteurs/internautes deviennent des partenaires virtuels et participent en tant qu'élément essentiel dans le flux de l'histoire. Notons qu'avec le développement constant du numérique, que ce soit via des applications pour smartphone ou via Internet, les histoires interactives continuent de se répandre. Ces récits se composent de narrations qui engagent les lecteurs au niveau de la structure de l'histoire et même de la narration. Nous citons par exemple Lifeline de Dave Justus (2015), un roman interactif sur smartphone disponible en anglais, en français et en allemand (figure 6). Dans cette histoire, le lecteur interagit avec un personnage fictif (le premier épisode est un astronaute perdu dans l'espace, le deuxième épisode est un jeune magicien) qui demande de l'aide (figure 7) et propose des suggestions pour son exploration (figure 8).

(La lecture numérique entre l'interactivité ...)Dr. Dalia Metawe 


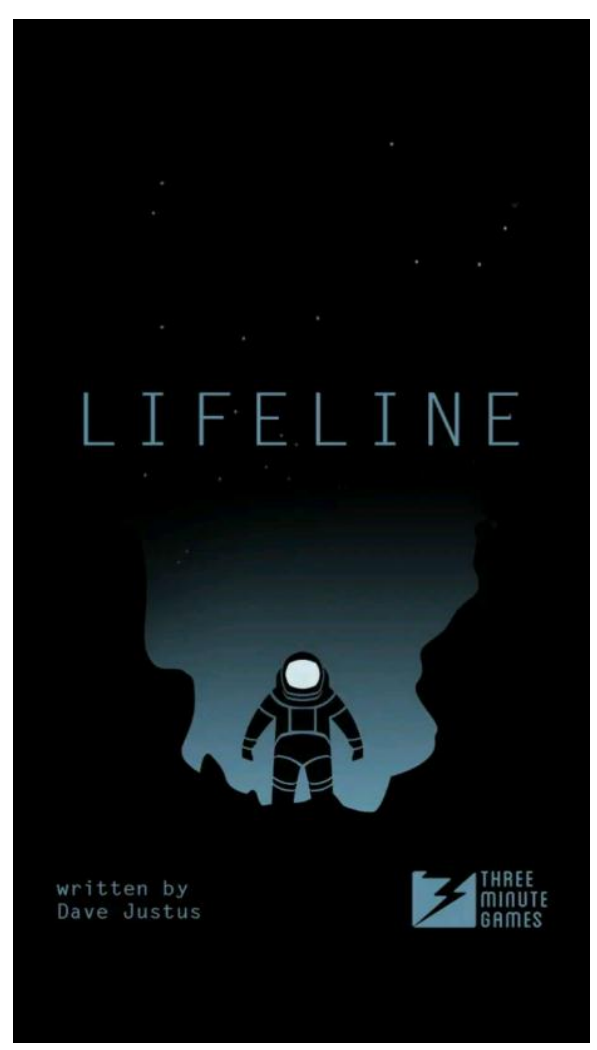

Figure (6) : capture-écran, Lifeline de Dave Justus (2015) 


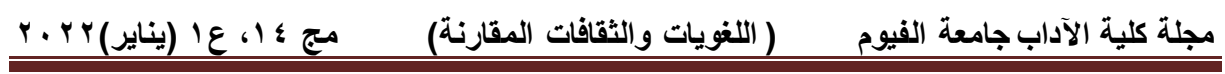

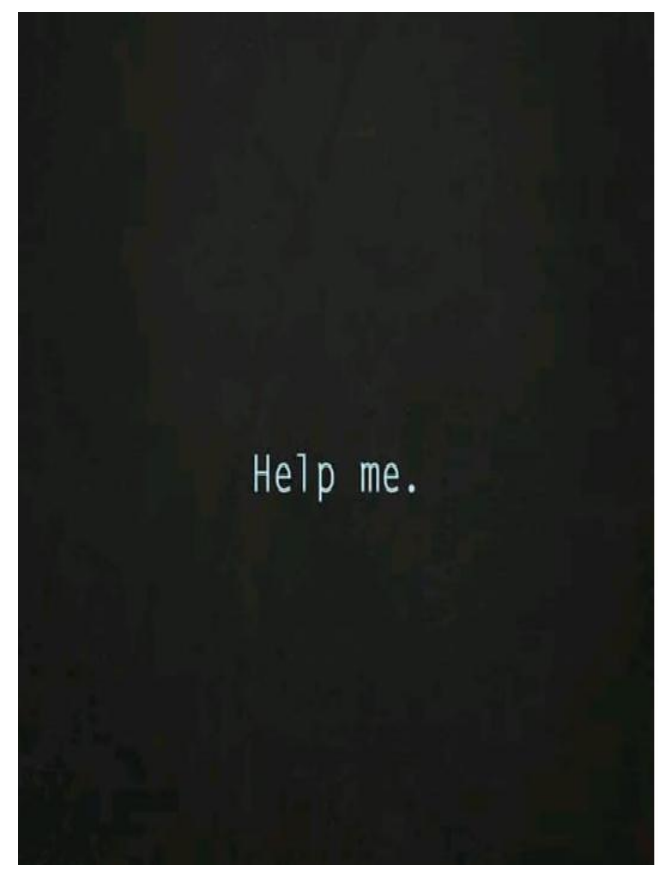

Figure (7) : capture-écran, Lifeline de Dave Justus (2015) 


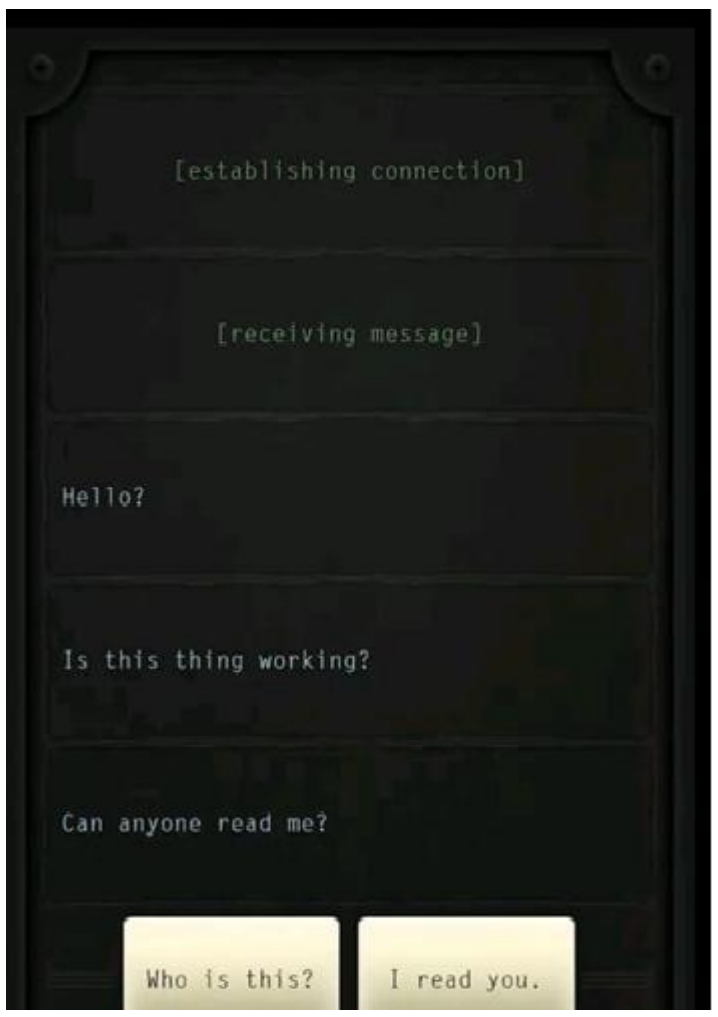

Figure (8) : capture-écran, Lifeline de Dave Justus (2015)

Ainsi la rencontre entre l'ordinateur et le lecteur produit de nouveaux modèles de discours, qui sont dérivés de la sémiotique du mouvement et de la manipulation, ce qui modifie considérablement l'activité de lecture sur écran. Remarquons que dans le processus particulier de lecture numérique, l'écran est le seul lieu d'interaction avec l'œuvre, il joue donc un rôle essentiel. Quant aux lecteurs/internautes, ils doivent avoir les compétences et les connaissances nécessaires pour établir de nouveaux repères afin d'identifier les œuvres et de se souvenir des passages importants et des expériences spécifiques lors de la

(La lecture numérique entre l'interactivité ...)Dr. Dalia Metawe 
lecture sur écran. La remise en question de la conception même du texte est certes très importante, car ce n'est plus l'ouvrage imprimé figé qui fournit au lecteur un texte à travers quelques pages, mais c'est le lecteur/interacteur qui constitue lui-même le texte par sa manière de naviguer dans l'espace fourni par l'ordinateur et par son interactivité ( $C f$., C. Bélisle \& A.V. Cuyck, 2004: 91).

Dans cette modalité de lecture numérique, la subjectivité du lecteur/internaute est constamment sollicitée par les dispositifs hypermédiatiques et interactifs: qu'il s'agisse de lui demander d'agir directement sur l'écran, ou de l'inviter à investir sa propre expérience pour comprendre le lien entre textes, images et animations en termes d'interactivité. Le lecteur se situe de ce fait toujours au centre de la communication avec l'œuvre, avec sa réception singulière et ses émotions. Pour cela, il doit maintenir une compréhension tacite avec le dispositif hypermédiatique pour pouvoir participer physiquement et intellectuellement à l'action narrative. En outre, avec l'évolution continue du monde numérique, des outils tels que les carnets numériques ou les blogs de lecture, équivalents virtuels des carnets et des journaux de lecture, sont considérés comme des outils privilégiés pour observer la réception singulière de l'œuvre littéraire. Ils peuvent promouvoir davantage l'expression de la subjectivité, grâce à des

(La lecture numérique entre l'interactivité ...)Dr. Dalia Metawe 
outils multimodaux, et suivre la voix et la perception intime des lecteurs/internaute ( $C f$., Murielle Szac).

\section{IV.La manipulation sur internet et la gestualité du} $\underline{\text { lecteur/utilisateur }}$

La manipulation sur internet et la gestualité du lecteur ne peuvent être comprises qu'en lisant sur l'écran de l'ordinateur, de la tablette, du smartphone, etc. Théoriquement, le numérique est caractérisé par la manipulation de systèmes unitaires discrets indépendants les uns des autres à travers des règles algorithmique formelles. La manipulation sur Internet se fait donc de deux manières: la numérisation constitue le nombre d'unités discrètes; l'informatisation consiste à les rendre manipulables par un ordinateur. Si le calcul permet de manipuler l'inscription sous la forme enregistrée, il peut également offrir à l'utilisateur la possibilité de manipuler l'inscription ( $C f$. Bouchardon, 2008). Par conséquent, les lecteurs peuvent utiliser le curseur de la souris pour manipuler le contenu à l'écran, déplacer des éléments, activer des liens, insérer leur propre texte dans le texte de l'histoire elle-même, ou introduire leur nom ou photo à travers une webcam. Afin d'effectuer ces gestes, le corps est fortement sollicité.

Serge Bouchardon a proposé, à cet égard, l'existence d'une «gestualité spécifique au numérique »(2012 b : 79). Il estime que la manipulation du lecteur/internaute est un «énoncé de gestes »

(La lecture numérique entre l'interactivité ...)Dr. Dalia Metawe 
(par exemple “cliquer" ou “déplacer la souris"), qui prend luimême une signification plus globale en fonction d'un contexte et d'un processus. Par ailleurs, Bouchardon a insisté à plusieurs reprises sur le rôle de la «manipulation gestuelle» dans la construction du sens de la création numérique (2009; 2011 : 38 ; 2018). Il évoque à ce propos le concept de «geste interfacé » de Jean-Louis Weissberg (Weissberg, 2006) pour illustrer l'idée selon laquelle les gestes manipulateurs affectent la construction du sens de l'œuvre numérique. Bouchardon a proposé un tableau synthétique de ces «énoncés de gestes » avec leurs «traits signifiants possibles », qui peuvent être combinés avec du texte ou des images (2011:42). Il pense que ce tableau de gestes et de leurs significations possibles pourrait être utile tant aux auteurs qu'aux lecteurs de création interactive :

«Dans la manipulation de créations interactives en ligne, nous avons affaire à des gestes différents : cliquer, doublecliquer, cliquer droit (PC), déplacer la souris (ou bouger le doigt sur le touchpad), laisser le doigt appuyé, relâcher le doigt, taper sur une touche du clavier, mais aussi parfois souffler dans un micro, bouger la tête devant une webcam » (Bouchardon, 2012 b : 79-80).

Néanmoins, il affirme qu'une difficulté est survenue : ces gestes sont en constante évolution, et il est difficile d'établir une liste stable de gestes. Par exemple, le pavé tactile d'un ordinateur

(La lecture numérique entre l'interactivité ...)Dr. Dalia Metawe 
récent permet à l'internaute d'utiliser deux doigts pour effectuer un zoom, un défilement et même une rotation d'impression. Ces gestes dépendent ainsi essentiellement de l'appareil.

Dans cette perspective, Yves Jeanneret a expliqué la différence entre la manipulation du livre papier et de l'œuvre numérique en rappelant que tourner les pages du livre ne présume pas a priori une interprétation spécifique du texte. En revanche, dans une œuvre interactive, cliquer sur un mot (Hypermot) ou un pictogramme (icône) lui-même est un acte d'interprétation (2000: 112-113). Il a affirmé que les manipulations interactives consistent avant tout en une «interprétation actualisée dans un geste » (ibid.: 121). La technologie numérique met ainsi à la disposition de lecteurs/usagers des prises matérielles afin qu'ils puissent contrôler certains éléments de l'appareil. Les lecteurs/internautes manipulent ces différents supports à l'écran en interagissant avec la matérialité du numérique, et grâce à ces manipulations, ils peuvent trouver l'interprétation correcte de l'œuvre et sa signification. Ces gestes manipulateurs font donc partie intégrante de l'œuvre interactive, sans ces gestes l'œuvre n'existerait pas.

Nicole Pignier et Benoît Drouillat, de leur part, ont confirmé l'importance des gestes manipulateurs dans les sémios du texte numérique. Pour analyser le rôle des gestes dans les sites

(La lecture numérique entre l'interactivité ...)Dr. Dalia Metawe 
web commerciaux et l'art numérique, les deux chercheurs ont qualifié ces signes dans le discours numérique de « signes iconiques ", dans lesquels une relation réciproque s'établit entre les actions des utilisateurs, leurs gestes et le contenu interactif à l'écran (par exemple, une image). Ils ont également souligné que l'auteur du discours numérique crée des effets de sens particuliers à travers «l'ethos », pour guider les lecteurs dans l'interprétation du texte. En d'autres termes, l'auteur fournit des outils de compréhension afin que chaque site puisse établir la manière dont le contenu organisationnel enracine la situation de communication dans la mémoire culturelle, que le « designer » active en tant que cadre pratique essentiel pour le parcours des lecteurs.

Pour illustrer l'importance de ces gestes manipulateurs dans la création numérique littéraire, prenons un exemple, Déprise de Serge Bouchardon et Vincent Volckaert, un récit numérique interactif parue en 2010 pour la version Flash, 2018 pour la version JavaScript et comme application pour smartphone en 2019. C'est l'histoire d'un homme qui sent qu'il a perdu le contrôle de sa vie. Cette œuvre numérique offre aux lecteurs/interacteurs plusieurs types de manipulations gestuelles : dessiner des bulles sur l'écran en déplaçant le curseur (figure 9), cliquer sur le texte pour lire entre les lignes (figure 10), glisser le curseur sur l'écran pour dévoiler l'image de la femme du

(La lecture numérique entre l'interactivité ...)Dr. Dalia Metawe 


\begin{tabular}{|c|c|c|}
\hline 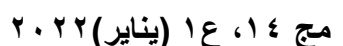 & (اللغويات والثقافات المقارنة) & مجلة كلية الآداب جامعة الفيوم \\
\hline
\end{tabular}

narrateur (figure 11), utiliser le curseur en essayant de contrôler les lettres pour trouver le discours du narrateur (figure 12), taper un texte à l'aide du clavier (figure 13).

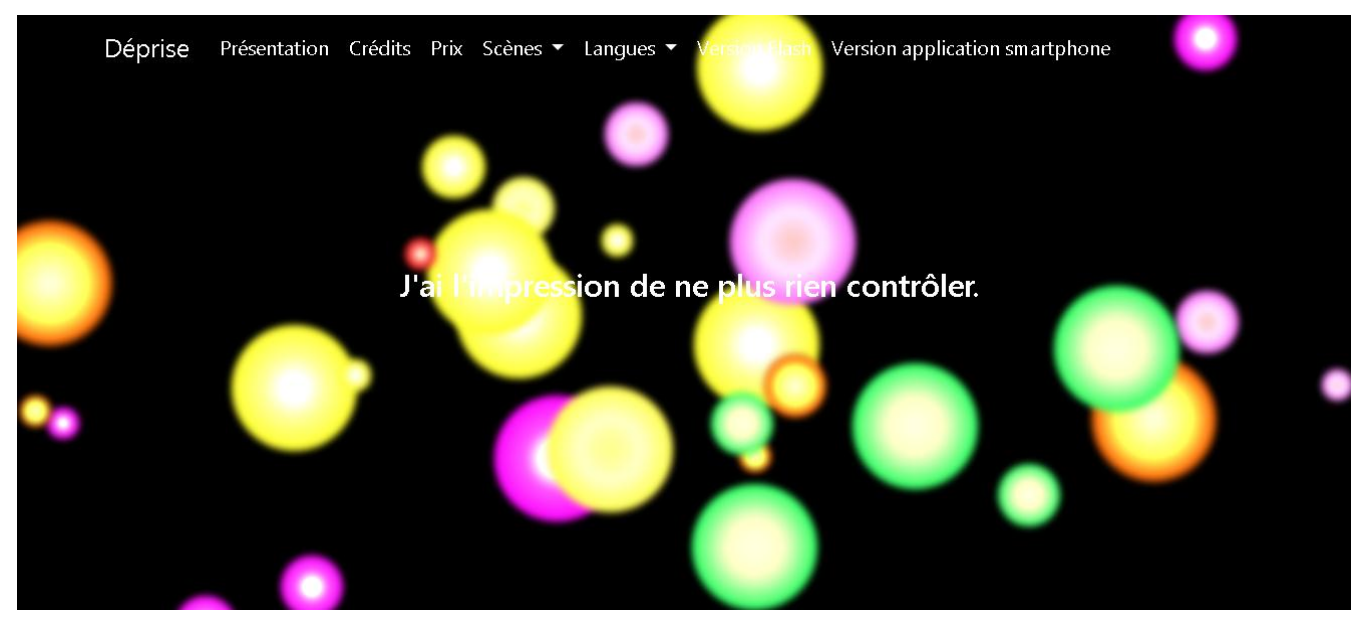

Figure (9) : capture-écran, Déprise de S. Bouchardon et V.

Volckaert (2010)

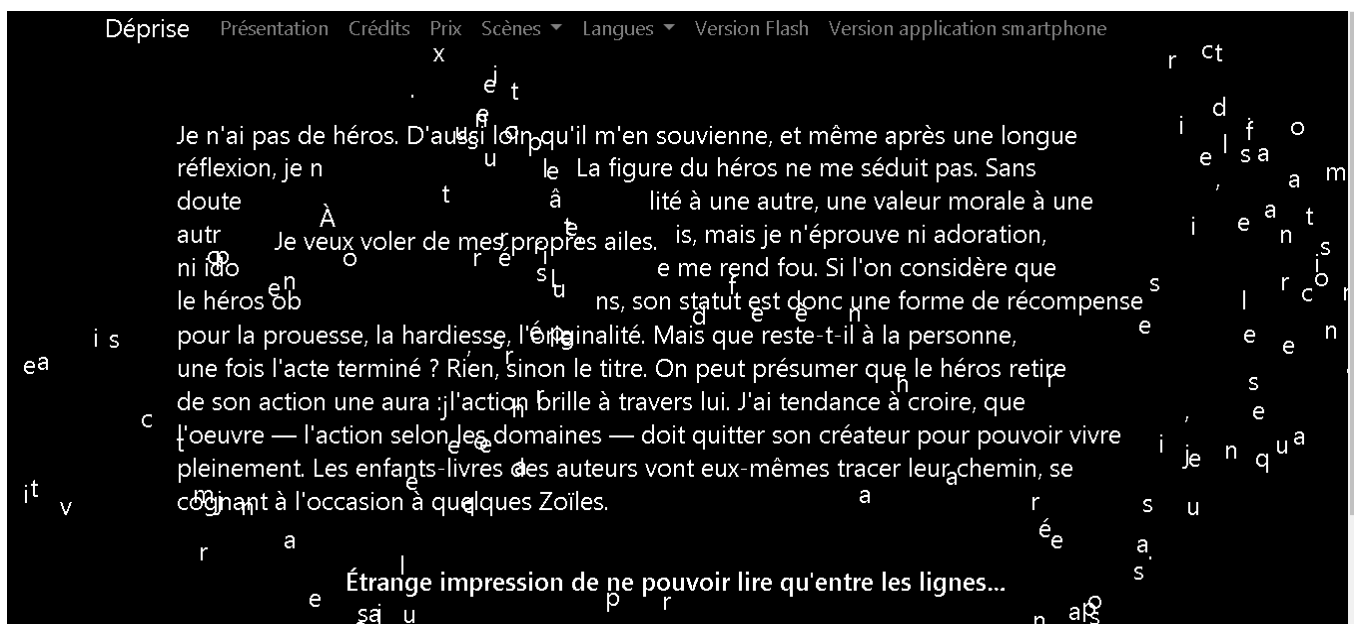

Figure (10) : capture-écran, Déprise de S. Bouchardon et V.

Volckaert (2010)

(La lecture numérique entre l'interactivité ...)Dr. Dalia Metawe 


\begin{tabular}{|c|c|c|}
\hline 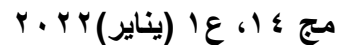 & ( اللغويات والثقافات المقارنة) & مجلة كلية الآداب جامعة الفيوم \\
\hline
\end{tabular}

Déprise Présentation Crédits Prix Scènes - Langues - Version Flash Version application smartphone

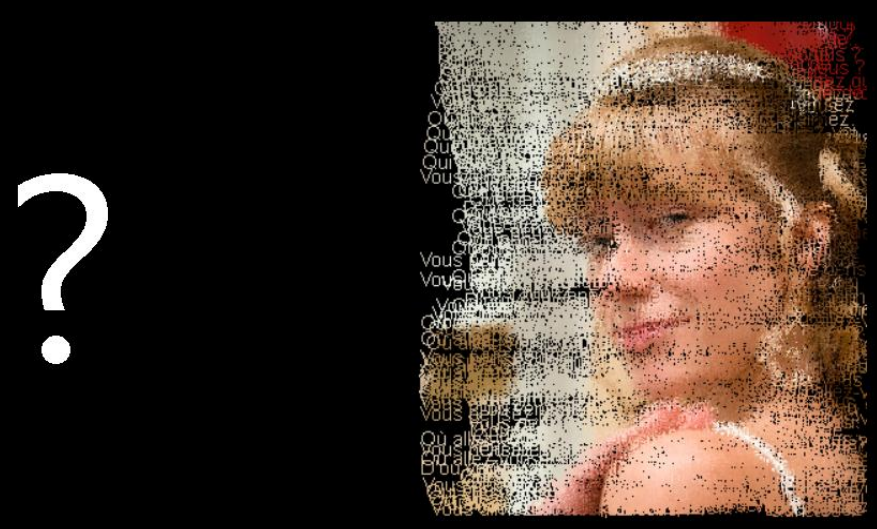

Qui d'elle ou moi suit l'autre?

Figure (11) : capture-écran, Déprise de S. Bouchardon et V.

Volckaert (2010)

Déprise Présentation Crédits Prix Scènes $\mathbf{V}$ Langues $\boldsymbol{V}$ Version Flash Version application smartphone

Retrouver une direction.

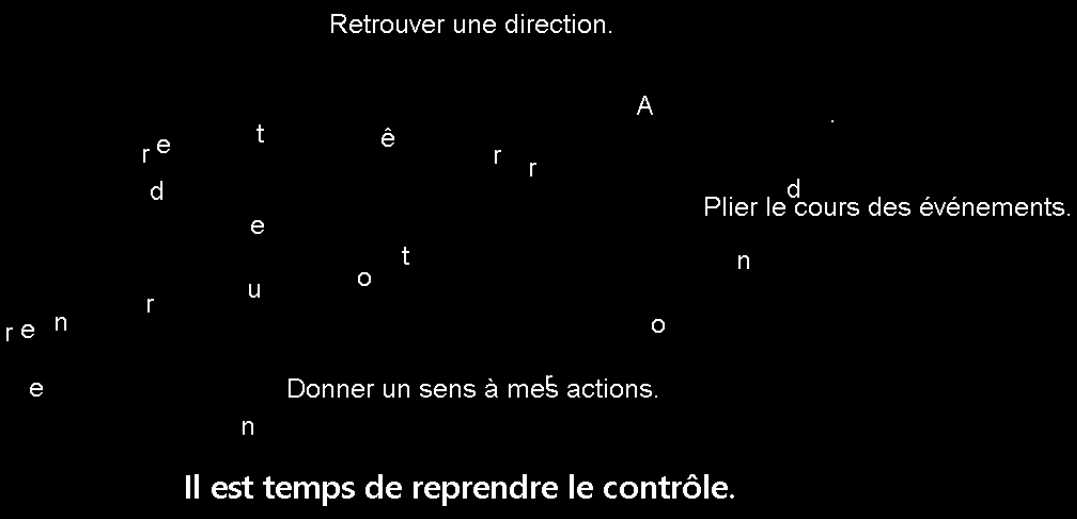

Figure (12) : capture-écran, Déprise de S. Bouchardon et V.

Volckaert (2010)

(La lecture numérique entre l'interactivité ...)Dr. Dalia Metawe 


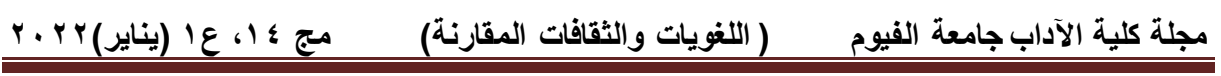

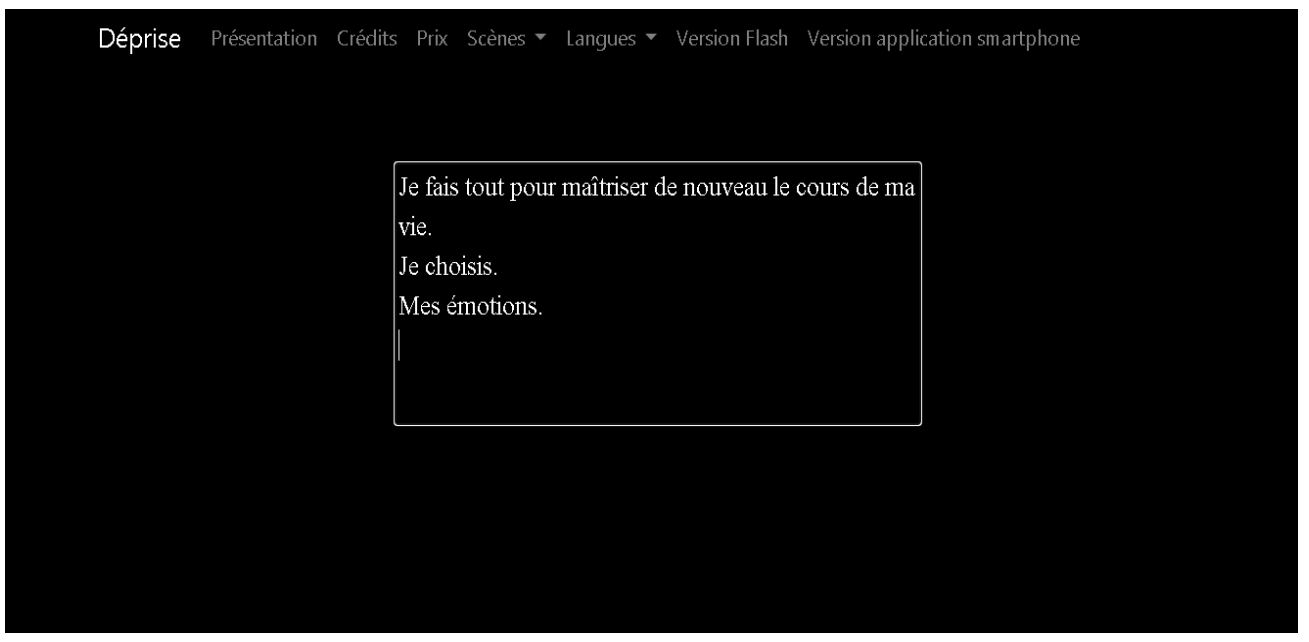

Figure (13) : capture-écran, Déprise de S. Bouchardon et V.

Volckaert (2010)

Dans cette histoire numérique, le lecteur doit faire face à la manipulation gestuelle, qui repose sur l'écart entre ses attentes lors de la manipulation et l'affichage vu à l'écran. Par conséquent, il interagit avec le sentiment de perte du narrateur et ses tentatives de maitriser de nouveau le cours de sa vie. Ainsi, dans la scène 3 , le narrateur essaie de comprendre un mot laissé par sa femme : est-ce un mot d'amour ou de rupture ? Le lecteur expérimente cette compréhension incertaine par des gestes de manipulations : si le lecteur/internaute déplace le curseur de la souris d'un côté de l'écran à l'autre, l'organisation des phrases sera inversée, et le mot d'amour devient un mot de rupture (figures 14, 15). La musique qui y est sélectionnée (de Carmen de Bizet) est également jouée à l'envers)

(La lecture numérique entre l'interactivité ...)Dr. Dalia Metawe 


\begin{tabular}{|c|c|c|}
\hline 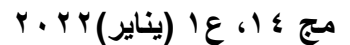 & ( اللغويات والثقافات المقارنة) & مجلة كلية الآداب جامعة الفيوم \\
\hline
\end{tabular}

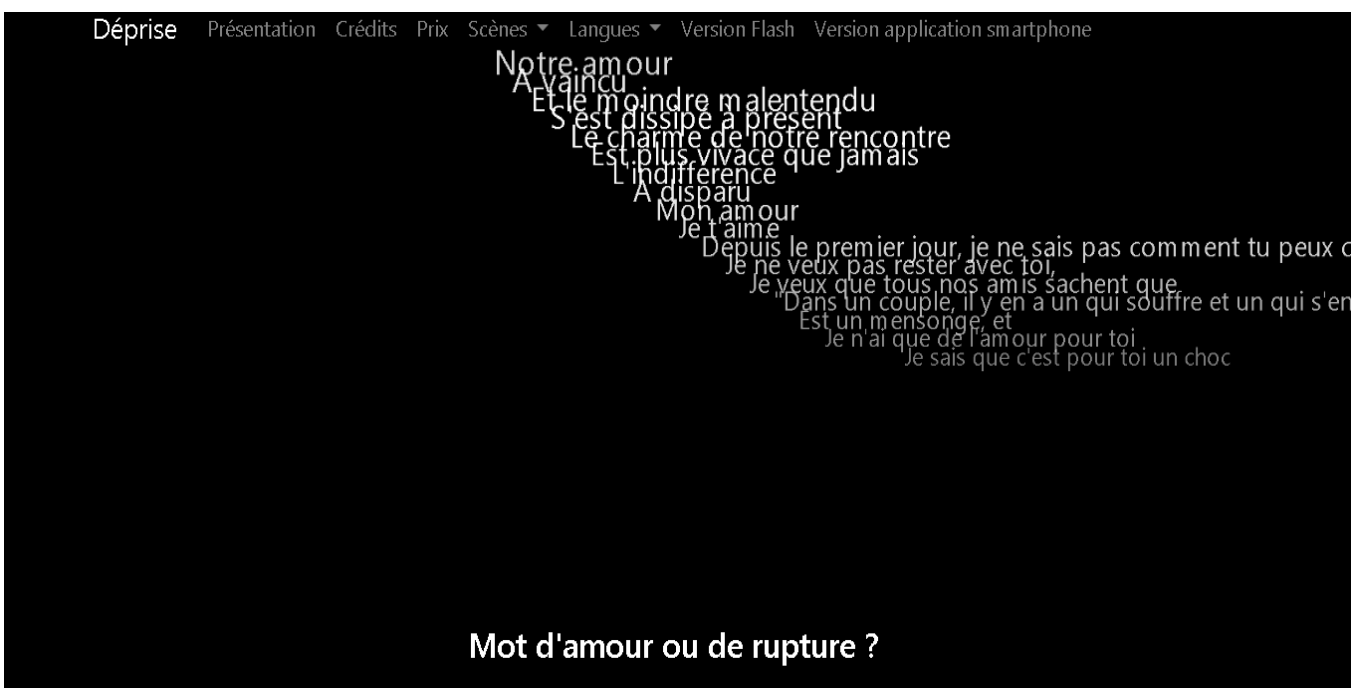

Figure (14) : capture-écran, Déprise de Bouchardon et Volckaert (2010), scène 3.

Déprise Présentation Crédits Prix Scènes - Langues - Version Flash Version application smartphone

Je sais que c'est pour toi un choc

Je n'ai que de l'amour pour toi

Est un mensonge, et

"Dans un couple, il y en a un qui souffre et un qui s'ennui Je veux que tous nos am is sachent que

Je ne veux pas rester avec toi,

Depuis le premier jour, je ne sais pas comment tu peux cro Je t'aime

Mon awe puis-je faire?

A disparu

Figure (15) : capture-écran, Déprise de Bouchardon et Volckaert (2010), scène 3.

Notons que dans cet exemple, la matérialité du texte numérique stimule les activités gestuelles des lecteurs/interacteurs. Elle est

(La lecture numérique entre l'interactivité ...)Dr. Dalia Metawe 
donc indissociable de l'acte de lecture numérique. Selon ce point de vue, S. Bouchardon, en montrant divers exemples (texte activé ou exploité par le lecteur, texte-matériau, texte constituant l'espace du mouvement), met en évidence : «[...] C'est le geste du lecteur qui révèle la matérialité du texte » (Bouchardon, 2008 : 138). Il explique dans un autre article que les gestes et le texte manipulable ne peuvent exister que lorsqu'il existe une connexion entre eux : cette relation constitue ensemble gestes et texte manipulable ( $C f$., Bouchardon, $2011: 38)$.

D'un point de vu esthétique, la poéticité de la création numérique provient de la relation entre les gestes du lecteur et le texte à manipuler, de sorte que les auteurs satisfassent dans une certaine mesure les attentes du lecteur. Dans cette perspective, Bouchardon, Saemmer et d'autres chercheurs comme Jean Clément mettent en évidence que la littérarité de ce genre du texte naît de la «défamiliarisation linguistique, iconique, sonore et gestuelle» avec la zone d'attente des lecteurs. Bouchardon indique que dans la littérature, la «défamiliarisation» implique le langage, c'est-à-dire l'aspect linguistique. Dans la littérature numérique, la «défamiliarisation » implique non seulement des aspects linguistiques, mais aussi des aspects iconiques et vocaux ainsi que des gestes. Il ajoute que lorsque les types de gestes dans les médias numériques (PC et supports tactiles) commencent à se stabiliser, la «défamiliarisation» peut être la

(La lecture numérique entre l'interactivité ...)Dr. Dalia Metawe 
plus claire. Alors ce que nous pouvons voir, c'est la tension entre la réflexion pour révéler le sens et les activités qui agissent pour l'effectuer ( $C f$., Bouchardon, 2018). Ainsi, les œuvres littéraires numériques sont principalement basées sur des dispositifs dans lesquels les lecteurs agissent, composent, construisent. Le programme fonctionne et gère des phénomènes éventuels, permettant aux lecteurs d'envisager des suggestions imprévisibles. Outre les nouvelles figures discursives et sémiotiques qui constituent dans une large mesure l'esthétique de la littérature numérique, l'esthétique de cette création littéraire est essentiellement issue de ce paysage numérique où la programmation et l'activité gestuelle des lecteurs jouent un rôle indispensable dans la révélation du sens de l'œuvre ( $C f$. J. Clément, $2011: 67)$.

Comme nous avons déjà montré, la production de littérature numérique n'est pas fondée seulement sur des mots, mais aussi sur des manipulations gestuelles et sur de l'animation (des mouvements). La nature de cette création hypermédiatique propose généralement un mode de lecture basé sur les gestes, «une lecture gestualisée » pour reprendre l'expression de Bouchardon. Cette fiction numérique n'exploite pas seulement les potentiels du numérique, mais elle propose des manipulations gestuelles des lecteurs/interacteurs, fournissant ainsi une «dimension performative » à l'activité de lecture en ligne

(La lecture numérique entre l'interactivité ...)Dr. Dalia Metawe 
(Bouchardon, 2007 : 110). Lorsque le corps du lecteur est présent dans l'œuvre, la lecture gestualisée devient une performance ou un jeu.

Cet aspect dynamique de la lecture sur écran permet à Jean Clément d'établir un lien intéressant entre les jeux et la littérature. Selon lui la littérature numérique relève du jeu, jeu qu'il se place même au cœur de cette littérature : "Cette dimension de jeu entre un auteur et un lecteur à travers la médiation d'une machine semble constitutive de la littérature numérique » (2011 : 93). Pour sa part, Lucie de Boutiny affirmait, lors d'un entretien du NEF à propos de son roman multimédia NON-roman, paru en feuilleton en ligne en septembre $1997:$ : «...] l'écrit à l'écran s'apparente à un jeu et non seulement se lit mais aussi se regarde ». Guidé par l'écrivain, les lecteurs/interacteurs peuvent faire des choix, prendre des décisions et exercer leur liberté en lisant, en apprenant progressivement des œuvres, comme les jeux vidéo: «Le lecteur va jouer l'œuvre, à sa façon à lui, ce qui lui donne caractère de performance, c'est une lecture qui devient unique » (C. Henry, $2018: 42$ )

Dans ce paysage numérique, l'acte de lecture devient par ailleurs de plus en plus «instrumenté ». Les œuvres électroniques ne sont pas seulement un support, mais aussi un outil qui peut améliorer la capacité des lecteurs à utiliser les moteurs de recherche pour rechercher, à intégrer des

(La lecture numérique entre l'interactivité ...)Dr. Dalia Metawe 
dictionnaires pour la connaissance et à utiliser des outils d'analyse complémentaire et facilement accessible via des hyperliens pour une meilleure compréhension. Il ne s'agit donc pas d'un moyen de reproduire la représentation familière du papier, mais d'un outil pratique qui peut promouvoir une lecture active et riche d'une manière que tout le monde peut utiliser, et constitue une porte d'entrée vers la culture (C. Bélisle \& A. v. Cuyck, 2004 : 96). N. Pignier et B. Drouillat indiquent à cet égard l'importance culturelle de la lecture numérique, soulignant que chaque créateur, chaque marque et chaque annonceur présente son propre « ethos » à travers l'interface de ses pages-écrans, et invite chaque lecteur/usager à partager un ensemble de valeurs, de traits de caractères, de système de sensibilité sociale au cours de son processus de lecture numérique (2008).

Avec le développement continu de la technologie numérique, certaines œuvres numériques peuvent introduire l'espace physique du lecteur/internaute dans la fiction, autrement dit la réalité spatiale du lecteur en ajoutant une dimension ludique puissante basée sur un rapport entre narrativité et spatialité, y compris la spatialité et la temporalité du lecteur/internaute. Citons à titre d'exemple The Cartographer's Confession de James Attlee (2017), une application pour smartphone qui raconte l'histoire de deux réfugiés après la seconde guerre mondiale. Ce roman interactif oblige les lecteurs à errer dans les rues de Londres et à

(La lecture numérique entre l'interactivité ...)Dr. Dalia Metawe 


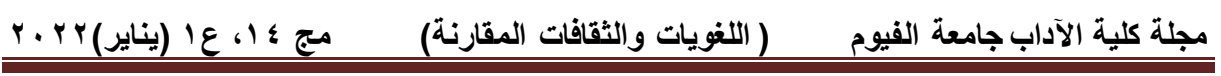

faire partie de l'histoire (figure 16). Ces romans hypermédias soulèvent la question de la relation entre le récit et l'espace, en particulier la relation entre l'espace physique de l'auteur et du lecteur couplé à des bases de données. Ainsi, le temps et l'espace réels du lecteur nourrissent la dimension ludique et dynamique dans la fiction numérique en brouillant la frontière entre réalité et fiction sur support numérique.

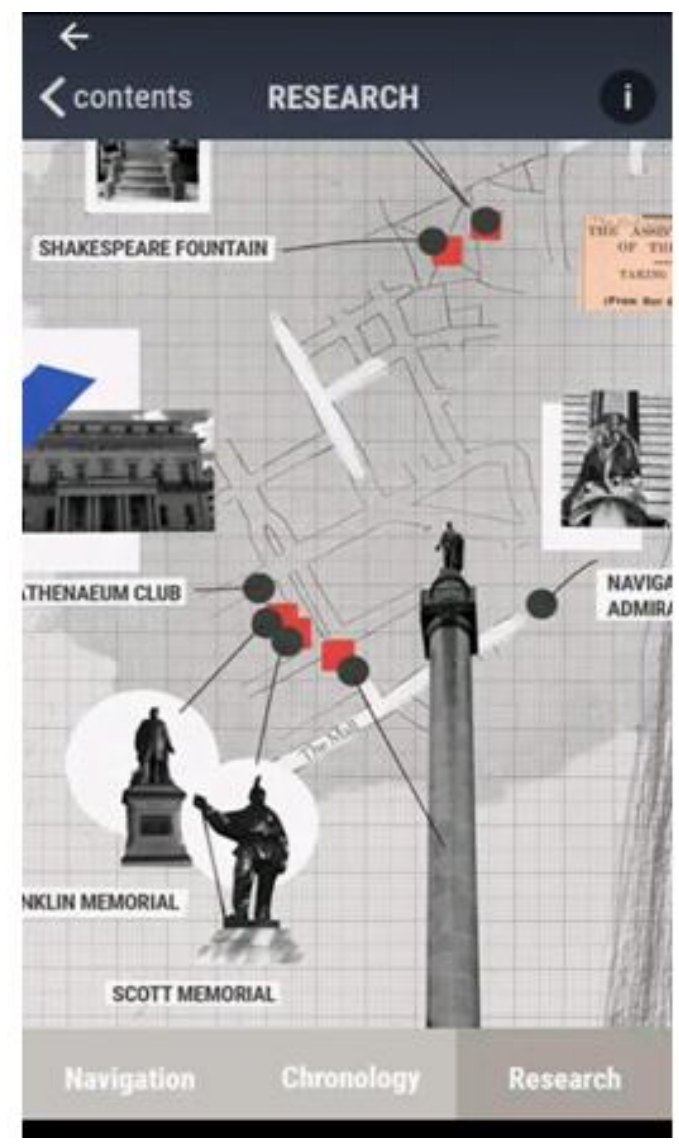

Figure (16) : capture-écran, The Cartographer's Confession de James Attlee (2017)

(La lecture numérique entre l'interactivité ...)Dr. Dalia Metawe 
Cette méthode de traitement de texte et d'autres formes sémiotiques ouvre ainsi d'énormes possibilités de création numérique interactive. La lecture sur Internet devient une activité nomade, fragmentée et de courte durée, naviguant d'hyperlien en hyperlien dans un ordre individuellement choisi. La littérature numérique expérimente des textes bifurqués, multimédias, interactifs, dans lesquels l'unité n'est jamais donnée, mais toujours construite et remise en question ( $C f$. Bouchardon, 2018).

\section{La dimension discursive de la lecture numérique}

La dimension discursive constitue l'un des aspects importants de la lecture numérique. Cette dimension met en relief la relation entre l'auteur et le lecteur ainsi que les activités collectives et collaboratives de la littérature numérique. L'informatique offre en effet aux auteurs la possibilité de communiquer avec les lecteurs via différentes plateformes numériques et réseaux sociaux. Grâce à ces communications en ligne, les lecteurs/internautes peuvent collaborer avec les auteurs au processus de création d'œuvres : par exemple, des écrivains comme François Bon et Cécile Portier mènent des ateliers d'écriture en ligne pour créer leurs œuvres numériques devant des lecteurs/internautes qui peuvent participer au développement de l'œuvre à travers des idées, des suggestions et des commentaires.

(La lecture numérique entre l'interactivité ...)Dr. Dalia Metawe 
Par ailleurs, les activités de lecture numérique font généralement l'objet d'expérimentations. S. Bouchardon propose le terme «lecture collective » qui fait référence à la projection d'un texte à défilement automatique sur un grand écran où les lecteurs peuvent se réunir (Bouchardon, 2007). Ces séances de «lecture collective » permettent aux lecteurs de se rencontrer et de partager une expérience de lecture commune. Selon Bouchardon, ce type de communication entre individus et collectifs établit, «un moyen de rêve commun », et met ainsi en évidence la dimension sociale de cette pratique en ligne (Ibid.). Les séances de lecture collective peuvent être organisées dans des endroits spécifiques ou en ligne, et les lecteurs peuvent lire le même texte à différents lieux en même temps. Au cours de ces rencontres, les lecteurs peuvent montrer leurs propres œuvres pour une lecture collective.

Nous avons donc un nouveau statut du lecteur/interacteur sur support numérique. Le terme «lect-acteur» que l'on retrouve chez des théoriciens comme Jean-Louis Weissberg (1999) ou Serge Bouchardon (2007) met en relief ce nouveau statut acquis par les lecteurs d'œuvres numériques. Selon Bouchardon, la «lect-acteur» est une sorte de lecture, considérée comme «coproduction» de l'environnement informatique, une sorte de lecture «gestuelle» et aussi une sorte de lecture-performance. Elle est aussi une lecture qui oscille entre les jeux et les

(La lecture numérique entre l'interactivité ...)Dr. Dalia Metawe 
expérimentations, inhérente à la «double lecture » ou à la «métalecture»(2007). On se réfère parfois au néologisme « lectant ", qui met l'accent sur la part active de la lecture numérique de manière plus subtile à travers le participe présent. Notons que l'intérêt de l'auteur pour les activités de lecture n'est pas seulement la volonté de passer le relais aux lecteurs, mais aussi une question importante du lien mutuel entre le processus d'écriture et de lecture. Néanmoins, Bouchardon affirme qu'en plus d'expérimenter des activités de lecture partagées, la lecture elle-même peut également être considérée comme une expérimentation dans les cuvres. D'une part, le lecteur pense que l'œuvre est un objet expérimentable et, d'autre part, l'auteur pense que l'espace du lecteur est un espace d'expérimentation (Ibid.).

À noter que l'ordre du livre imprimé favorise la diversité de lecture, d'interprétation, et forme une communauté d'apprentissage autour d'un corpus partagé. La révolution numérique a perturbé et dépassé cet ordre. Cela permet au texte de se déployer en dehors du livre et d'intégrer toute représentation dans une hypertextualité infinie, interactive et dynamique (générant ainsi du big data). Depuis lors, la lecture est devenue une méta-lecture, c'est-à-dire qu'elle n'est plus la lecture linéaire ou la lecture intensive d'un livre, mais l'auto-

(La lecture numérique entre l'interactivité ...)Dr. Dalia Metawe 
construction d'un parcours cognitif à travers diverses formes de lecture en constante transformation.

\section{Conclusion :}

L'émergence du Web 2.0 a apporté de réels changements dans les concepts de base de l'écriture littéraire et du texte, et ces changements ont complètement modifié l'acte de lecture sur support numérique. Grâce à cette technologie, les auteurs connectés à Internet peuvent créer des textes numériques mouvants et manipulables. En conséquence, de nouvelles formes littéraires hypermédias, dynamiques et multi-codes sont apparues en ligne. Cette production numérique hypermédiatique transforme la lecture en lecture dynamique, dans laquelle le lecteur joue un rôle majeur et participe dans une large mesure à la construction du sens de l'œurre.

L'hypertexte a modifié l'acte de lecture linéaire traditionnel en fournissant une méthode de lecture non-linéaire qui permet à chaque lecteur de suivre un parcours individuel selon son choix. Le texte hypertextuel dépasse la contrainte et la limite de la linéarité du texte imprimé et le sens imposé par la matérialité du livre papier. Dans ce contexte, les écrivains de l'hypertexte fictionnel créent des histoires en ligne selon double contrainte : non-linéarité et fragmentation de l'histoire. Par ailleurs, la dimension multimédia et l'interactivité du lecteur ont apporté de l'innovation à la lecture numérique. La dimension iconique (La lecture numérique entre l'interactivité ...)Dr. Dalia Metawe 
permet au lecteur de manipuler les éléments visuels et sonores qui apparaissent à l'écran, recréant ainsi une autre combinaison de texte à partir du matériel suggéré. Le texte numérique ne peut donc exercer son sens qu'à travers la manipulation du lecteur/interacteur à l'écran. L'interactivité constitue ainsi un élément indispensable de l'acte de lecture numérique basant sur la rencontre inattendue des processus informatiques avec les interventions des lecteurs/internautes dans ces processus. D'autre part, les manipulations gestuelles des lecteurs/interacteurs fournissent à l'activité de lecture en ligne une dimension performative, en d'autres termes, en présence du corps du lecteur dans la création numérique, la «lecture gestualisée » devient une performance ou un jeu. De plus, la réalité spatiale et temporelle du lecteur est introduite dans certaines œuvres numériques pour y ajouter une puissante dimension ludique qui se dessine à travers le rapport entre narrativité/spatialité et temporalité réelles du lecteur/internaute.

L'un des aspects importants de la lecture numérique est la dimension discursive qui met en relief la relation entre l'auteur et le lecteur ainsi que les activités collectives et collaboratives de la littérature numérique. L'informatique offre aux auteurs la possibilité de communiquer avec les lecteurs via différentes plateformes numériques et réseaux sociaux. Cette communication en ligne permet aux lecteurs de participer au

(La lecture numérique entre l'interactivité ...)Dr. Dalia Metawe 
processus de création d'œuvres avec les auteurs. Par ailleurs, les activités de lecture numérique font généralement l'objet d'expérimentations: les auteurs organisent des séances de «lecture collective » permettant aux lecteurs de se rencontrer et de partager une expérience de lecture commune. Ce type de communication entre individus et collectifs met en évidence la dimension sociale de cette pratique en ligne.

De ce qui précède, nous constatons que la rencontre active entre l'ordinateur et les auteurs a produit des nouveaux modèles de discours littéraire basés sur la sémiotique du mouvement et de la manipulation, modèles qui ont profondément modifié l'activité de lecture à l'écran. Ces œuvres numériques hypermédias s'expriment sous la forme d'une double communication : communiquer avec les lecteurs et communiquer avec elles-mêmes. Dans cette modalité de lecture numérique multimodale, le lecteur participe activement à la construction du sens de l'œuvre. La subjectivité du lecteur/internaute est constamment sollicitée par les dispositifs hypermédiatiques et interactifs. Par conséquent, le lecteur, sa réception, ses gestes, et ses émotions singulières sont toujours au centre de la communication avec l'œuvre.

(La lecture numérique entre l'interactivité ...)Dr. Dalia Metawe 


\section{Références :}

I. Articles de revue et chapitres d'ouvrages consacrés à la lecture numérique

- AUDET, René. BROUSSEAU, Simon. (2011). «Pour une poétique de la diffraction de l'œuvre littéraire numérique. L'archive, le texte et l'œuvre à l'estompe ». Protée. Vol. 39, $\mathrm{n}^{\mathrm{o}} 1$. Disponible en ligne: https://www.erudit.org/fr/revues/pr/2011-v39n1-r5004899/1006723ar/. Consulté le 14-6-2020.

- BELISLE, Claire. CUYCK, Alain Van. (2004). «Pratiques de lecture et livres électroniques ». La lecture numérique : réalités, enjeux et perspectives. C. Bélisle (dir.). Collection Référence. Presses de l'ENSSIB. Disponible en ligne : https://www.enssib.fr/bibliothequenumerique/documents/68264-la-lecture-numeriquerealites-enjeux-et-perspectives.pdf. Consulté le 2811-2020.

- BOOTZ, Philippe. (2011). «La littérature numérique en quelques repères ». Chapitre VI. Lire dans un monde numérique. Claire Bélisle (dir.). Presses de l'ENSSIB. pp. 206-253. Disponible en ligne: https://books.openedition.org/pressesenssib/1095. Consulté le 28-11-2020.

- BOUCHARDON, Serge. (2007). «Les œuvres de littérature numérique ». Chapitre III. Un laboratoire de littératures: littérature numérique et internet. Serge Bouchardon (dir.). Éditions de la Bibliothèque publique d'information. pp. 147-226. Disponible en ligne

(La lecture numérique entre l'interactivité ...)Dr. Dalia Metawe 
https://books.openedition.org/bibpompidou/232\#tocto 1n2. Consulté le 2/8/2020.

- BOUCHARDON, Serge. (2008). « La littérature numérique ». Terminal, 101.Disponible en ligne: http://journals.openedition.org/terminal/4472.

Consulté le 22/10/2020.

- BOUCHARDON, Serge. (2011). "Une esthétique de la matérialité ». Des figures de manipulation dans la création numérique. Protée, 39 (1). pp. 37-46. Disponible en ligne : https://doi.org/10.7202/1006725ar. Consulté le 4/3/2020.

- BOUCHARDON, Serge. (2012) (a). «Du récit hypertextuel au récit interactif ». Revue de la BNF. $\mathrm{n}^{\circ}$ 42. pp. 13-20. Disponible en ligne : https://www.cairn.info/revue-de-la-bibliothequenationale-de-france-2012-3-page-13.htm. Consulté le 1/7/2020.

- BOUCHARDON, Serge. (2012) (b). «Manipulation de médias à l'écran et construction du sens ». Médiation Et Information. Thierry Lancien (dir.). $\mathrm{n}^{\circ} 34$ (MEI). L'Harmattan. Paris. pp. 79-91. Disponible en ligne : https://www.researchgate.net/publication/270216561.

Consulté le 20/9/2020.

- BOUCHARDON, Serg. (2018). «Littérature numérique, 10 marches à franchir. Mind the gap! 10 gaps for Digital Literature? ». Disponible en ligne : http://www.utc.fr/ bouchard/Bouchardon-ELO18francais.pdf. Consulté le 1/12/2020.

- CHANDELIER, Emilie. (14 Mai 2019). «La lecture numérique, la lecture sur écran : que dit la recherche?».

(La lecture numérique entre l'interactivité ...)Dr. Dalia Metawe 
Disponible en ligne : https://pedagogie-numerique.acbesancon.fr/2019/05/la-lecture-numerique-la-lecturesur-ecran-que-dit-la-recherche/. Consulté le 4/6/2020

- CLEMENT, Jean. (2011). «Poétique du hasard et de l'aléatoire en littérature numérique ». Protée, 39 (1). pp. 67-76. Disponible en ligne: https://doi.org/10.7202/1006728ar. Consulté le 4/7/2020.

- DEBEAUX, Gaëlle. (2019). «Lignes de fuites poétiques du récit numérique ». Usages, formes et enjeux de la "poésie numérique ». Claire Chatelet et Gwendolyn Kergourlay (dir.). Komodo, vol. 12. Disponible en ligne : http://komodo21.fr/lignes-de-fuites-poetiquesrecit-numerique/. Consulté le 15/11/2020.

- GOLSON, Emily. (1999).« Cognition, Meaning and Creativity: On reading student hypertexts ». Contexts, Intertexts and Hypertexts. DeWitt S. L., Strasma K., Cresskill (éd.). Hampton Press. pp. 155- 175.

- ROUET, Jean-François. (16-17 Mars 2016). «Quelles sont les spécificités de la lecture numérique ?». Conférence de consensus, lire, comprendre, apprendre, Disponible en ligne: http: //www.cnesco.fr/wp-content/uploads/2018/04/14-Rouet.pdf. Consulté le 2/6/2020.

- SAEMMER, Alexandra. (2011). «Hypertexte et irradiation iconique ». Les Cahiers du numérique. Vol. 7, $\mathrm{n}^{\circ}$ 3-4. Caroline Angé (dir.). Lavoisier (Editeur). pp. 47-69.Disponible en ligne: https://www.cairn.info/revue-les-cahiers-dunumerique-2011-3-page-47.htm. Consulté le 2/8/2020. Consulté le 6/7/2020

(La lecture numérique entre l'interactivité ...)Dr. Dalia Metawe 
- SZAC, Murielle. (27 Février 2017). «L'apprentissage de la lecture littéraire peut s'appuyer sur les spécificités des textes numériques ». Disponible en ligne :http://www.bayardeducation.com/article/lapprentissage-de-la-lecture-litteraire-peut-s-appuyersur-les-specificites-des-textes-numeriques.html.

Consulté le 12-11-2020.

\section{Ouvrages consacrés à la littérature numérique}

- AARSETH, Espen J. (1997). Cybertext: Perspectives on Ergodic Literature. The Johns Hopkins University Press.

- BOUCHARDON, Serge. (2009). Littérature numérique : Le récit interactif. Hermès. Paris.

- BOUCHARDON, Serge. (2014). La valeur heuristique de la littérature numérique, Hermann, coll. «cultures numériques ». Paris. $\quad$ En ligne: http://www.costech.utc.fr/CahiersCOSTECH/IMG/pd f/bouchardon-hdr.pdf. Consulté le 19/11/2020.

- JEANNERET, Y. (2000).Y a-t-il vraiment des technologies de l'information?. Éditions universitaires du Septentrion. Paris.

- PIGNIER, Nicole, DROUILLAT, Benoît. (2008). Le webdesign : Sociale expérience des interfaces web. Sciences publications. Coll. Forme et sens. Hermès. Paris.

- SAEMMER, Alexandra.(2015). Rhétorique du texte numérique. Figures de la lecture, anticipations de pratiques. Villeurbanne. Coll. «Papiers». Presses de l'ENSSIB.

\section{Mémoire de master}

(La lecture numérique entre l'interactivité ...)Dr. Dalia Metawe 
- HENRY, Clara. (2018). L'archivage de la littérature numérique en ligne. Mémoire de Master, Sous la direction de Clément Oury. Université de Lyon. Disponible en ligne : https://www.enssib.fr/bibliothequenumerique/documents/68377-1-archivage-de-lalitterature-numerique-en-ligne.pdf. Consulté le 4/9/2020.

\section{Entretiens}

- LEBERT, Marie. DE BOUTINY, Lucie. Entretiens du $N E F$ - Lucie de Boutin. Disponible en ligne: http://barthes.enssib.fr/translatio/miroirnef/entretiens/boutiny.htm. Consulté le 12-11-2020.

\section{Euvres littéraires numériques}

- ATTLEE, James. (2017).The Cartographer's Confession. Fiction interactive sur smartphone. Consulté le $1 / 12 / 2020$.

- BALPE, Jean-Pierre. (Juillet 2020). Plus tard. Création numérique en ligne: http://www.balpe.name/Plustard. Consulté le 5/12/2020.

- BOUCHARDON, Serge. VOLCKAERT, Vincent. (2010). Déprise. Récit en ligne: https://bouchard.pers.utc.fr/deprise/home. Consulté le 2/3/2020.

- CHAMBEFORT, François. (2017). Lucette, gare de Clichy. En ligne: http://www.francoisechambefort.com/lucette-gare-de-clichy. Consulté le 10/11/2020.

- JUSTUS, Dave. (2015). Lifeline. Fiction interactive sur smartphone. Consulté le 1/12/2020.

(La lecture numérique entre l'interactivité ...)Dr. Dalia Metawe 


\section{القراءة الإكترونية بين التفاعلية والحركية} ملخص

يحث مفهوم القراءة التقليدي القراء على قراءة النص بطريقة منتظمة من البداية إلى النهاية. ولكن نتغير هذه الصورة عند القراءة على شاشة الكمبيوتز أو الجهاز اللوحي أو الهاتق المحمول. مع ظهور أجهزة الكمبيوتر متعددة الوسائط، وخاصة مع وجود الإنترنت، تغيرت خصائص وشكل النص الرقمي تغيرات أساسية، والنتي غيرت بدورها علاقتتا بالقراءة تمامًا. لقد أصبحت الرقمية أداة إبداعية نشطة وأسست علاقة متبادلة بين نظام تكنولوجيا المعلومات ومستخدميه. ونتيجة لذلك،أصبحت ممارسة القراءة الرقمبة،على مدى العقدين الماضيين ،اتجاها سائدا، لا سيما بين الثباب. في هذه الدراسة سنناقش طبيعة ممارسة القراءة ووضع القراء علي الوسائط الرقمية. سنشرح خصوصية القراءة الرقمية من خلال دراسة الفرق بينها وبين القراءة التقليدية في الكتب المطبوعة. سنقوم أيضًا بفحص ما إذا كانت هذه القراءة الرقمية لها أسلوبها الخاص بحيث يمكن للقراء التدخل في العمل الأدبي وما إذا كانت طريقة القراءة الجديدة عبر الإنتزنت تؤثز على عملية بناء معنى العمل.

كلمات مفتاحيه: القراءة الرقمية، الأسلوبية، التفاعلية، التعامل مع النص، الحركية. 